\title{
Non-linear Temperature Profiles
}

\author{
T. Ficker, J. Myslín, Z. Podešvová
}

Non-linear temperature profiles caused by temperature-dependent thermal conductivity $\lambda(\mathrm{T})$ of wall materials are discussed. Instead of conventional thermal resistance, modified effective resistance has been introduced.

Keywords: temperature profile in building structures, temperature-dependent thermal conductivity, Fourier's thermal laws.

\section{Introduction}

Heat conduction is the irreversible transport process that has been studied by non-equilibrium thermodynamics for very long time. It is often accompanied by the transport of mass (diffusion) and their corresponding transport equations are mutually coupled and form a system of equations. Nevertheless, in solids - to a certain extent - these two processes can be investigated separately. Heat conduction is governed by two Fourier laws, while diffusion is governed by, two Fick laws. Either group of laws is represented by differential equations of the same type so that their solutions are interchangeable if the initial and boundary conditions are also equivalent.

Fourier's first and second laws for heat flux $\vec{q}$ and temperature field $T(x, y, z, t)$ can be expressed as follows

$$
\begin{aligned}
& \vec{q}=-\lambda \nabla T, \\
& \rho c \frac{\partial T}{\partial t}=\nabla(\lambda \nabla T)+q^{*},
\end{aligned}
$$

where $\rho, c, \lambda$ and $q^{*}$ are density, heat capacity, thermal conductivity and volume heat flux of the sources, respectively. If there are no sources $q^{*}=0$, temperature field is independent on time $\frac{\partial \mathrm{T}}{\partial t}=0, \lambda$ is constant and heat flux is unidirectional (as is usual in thermal building technology), then the Fourier's laws (1), (2) read

$$
\begin{aligned}
& |\vec{q}|=q=-\lambda \frac{\mathrm{d} T(x)}{\mathrm{d} x}, \\
& \frac{\mathrm{d}^{2} T(x)}{\mathrm{d} x^{2}}=0 .
\end{aligned}
$$

For the boundary conditions $T(0)=T_{1}>T(d)=T_{2}$ the simple system of differential equations (3), (4) will yield the following solution

$$
\begin{aligned}
& q=\frac{T_{1}-T_{2}}{\frac{d}{\lambda}}, \\
& T(x)=T_{1}-\frac{T_{1}-T_{2}}{d} x .
\end{aligned}
$$

It is obvious that the heat flux $q$ is constant and the temperature field (profile) $T(x)$ inside the wall shows perfect linearity. This is the common situation described in all textbooks of building thermal technology for buildings and thermal standards many countries.

It follows from the preceding discussion that the first necessary condition for the linear temperature profile $T(x)$ is the existence of the steady thermal state which resulted from the time independence of the temperature profile

$$
\frac{\mathrm{d} T}{\mathrm{~d} t}=0 \Rightarrow q=\text { constant }
$$

and the second condition requires the thermal conductivity $\lambda$ to be a positive constant

$$
\frac{\mathrm{d} \lambda}{\mathrm{d} x}=0 \Rightarrow \lambda=\text { constant } .
$$

The positivity of $\lambda$ follows from its physical definition. If some of those conditions are not fulfilled a non-linear temperature profile $T(x)$ can appear. Frequently the non-linearity is caused by the dependence of $\lambda$ on temperature and moisture.

\section{Variable thermal conductivity}

Let us assume a dry material inside a wall whose thermal conductivity $\lambda$ depends only on temperature $T(x)$

$$
\frac{\mathrm{d} \lambda(T(x))}{\mathrm{d} x} \neq 0 \text {. }
$$

The temperature dependence of $\lambda$ is a common feature of all building materials and is often investigated especially with new materials. If a building material is used in a narrow temperature range (e. g. within several tens of degrees) the linear dependence

$$
\lambda=b T(x)+\lambda^{*}
$$

is a good approximation, as can be seen in Fig. 1. The linear graphs in Fig. 1 have been created by fitting the corresponding data [1] by the least-square method

$\lambda=2.98 \cdot 10^{-4} T+0.0525 \mathrm{Wm}^{-1} \mathrm{~K}^{-1}$ (Asbestos),

$\lambda=1.90 \cdot 10^{-4} T+0.0116 \mathrm{Wm}^{-1} \mathrm{~K}^{-1}$ (Foam concrete),

$\lambda=1.40 \cdot 10^{-4} T-0.0057 \mathrm{Wm}^{-1} \mathrm{~K}^{-1}$ (Cork),

$\lambda=1.10 \cdot 10^{-4} T-0.0034 \mathrm{Wm}^{-1} \mathrm{~K}^{-1}$ (Glass wool).

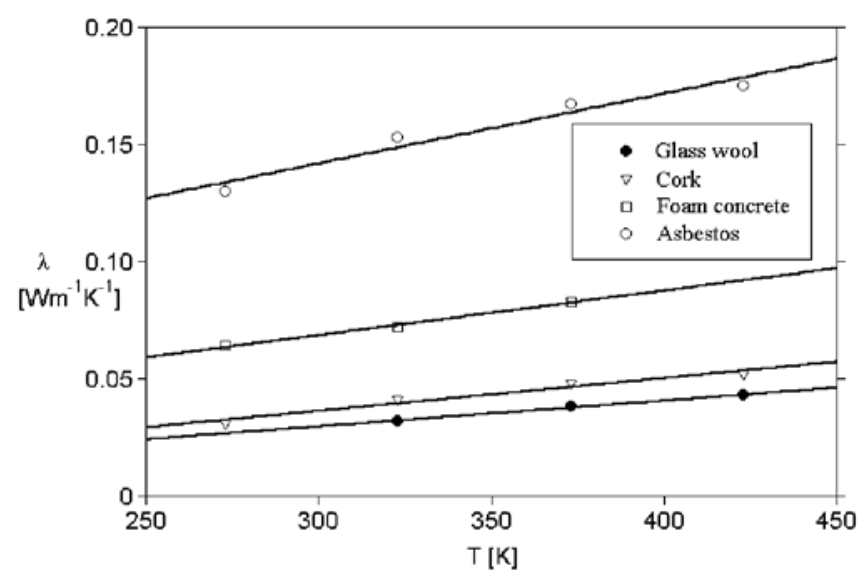

Fig. 1: Linear behaviour of thermal conductivity in a narrow temperature range 
Nevertheless, in a wider temperature range (several hundreds of degrees and more) a non-linear fit for $\lambda$ must be employed. Our experience shows that a reasonable approximation is provided with parabolic function

$$
\lambda-\lambda_{0}=a\left(T(x)-T_{0}\right)^{2}
$$

where $\lambda_{0}, a, T_{0}$ are parameters to be fitted. Fig. 2 illustrates the parabolic behaviour of $\lambda(T)$ for the same materials as given in Fig. 1 but in a much wider temperature range. The graphs in Fig. 2 have the following parameters

$\lambda-0.176=-1.5 \cdot 10^{-6}(T(x)-447)^{2} \mathrm{Wm}^{-1} \mathrm{~K}^{-1}$ (Asbestos),

$\lambda-0.057=6 \cdot 10^{-7}(T(x)-165)^{2} \mathrm{Wm}^{-1} \mathrm{~K}^{-1}$ (Foam concrete), (17)

$\lambda-0.053=-6 \cdot 10^{-7}(T(x)-465)^{2} \mathrm{Wm}^{-1} \mathrm{~K}^{-1}$ (Cork),

$\lambda-0.053=-2 \cdot 10^{-7}(T(x)-648)^{2} \mathrm{Wm}^{-1} \mathrm{~K}^{-1}$ (Glass wool).

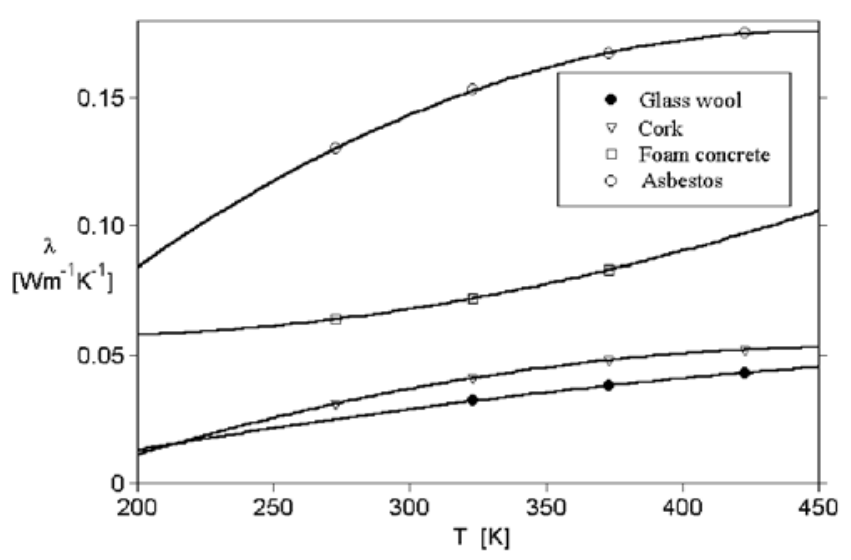

Fig. 2: Parabolic behaviour of thermal conductivity in a wider temperature range

Recently measurement of $\lambda(T)$ was published [2] for high performance concrete in a wide temperature interval ranging from $100^{\circ} \mathrm{C}$ to $800^{\circ} \mathrm{C}$. At first sight the graphs $\lambda(T)$ in [2] resemble a parabolic behaviour (15). For such pronounced non-linear behaviour of $\lambda(T)$ and for such wide temperature range, strong non-linearity of $T(x)$ must be expected.

The aim of this paper is to specify the non-linear character of temperature profile $T(x)$ either in the case of linear conduction function (10) or in the case of parabolic function (15).

\section{Non-linear temperature profiles}

Provided thermal conductivity $\lambda$ is not constant but a function of temperature $\lambda(T(x))$, Fourier's laws written in one dimension assume the following form when a steady state is reached

$$
\begin{aligned}
& q=-\lambda(T) \frac{\mathrm{d} T(x)}{\mathrm{d} x}, \\
& \frac{\mathrm{d}}{\mathrm{d} x}\left[\lambda(T) \frac{\mathrm{d} T(x)}{\mathrm{d} t}\right]=0 .
\end{aligned}
$$

Now the corresponding solution $[T(x), q]$ will depend not only on the boundary conditions $T(0)=T_{1}, T(d)=T_{2}$ but also on the form of the function $\lambda(T)$. Let us discuss both the mentioned non-linear dependencies $\lambda(T)$, i.e., the relations (10) and (15) from Chapter 2.

\subsection{Linear temperature dependence $\lambda(T)$}

Using the linear function (10) and solving the system of Eqs. (20), (21) for the usual boundary conditions $T(0)=T_{1}$, $T(d)=T_{2}$, it is possible to obtain

$$
\begin{aligned}
& q=\frac{T_{1}-T_{2}}{\frac{d}{\lambda^{*}}}+\frac{T_{1}^{2}-T_{2}^{2}}{2 \frac{d}{b}}=\frac{T_{1}-T_{2}}{R_{e f f}^{*}}, \\
& T(x)=\sqrt{\left(\frac{\lambda^{*}}{b}+T_{1}\right)^{2}-\left[\frac{1}{d}\left(T_{1}^{2}-T_{2}^{2}\right)+\frac{2 \lambda^{*}}{b d}\left(T_{1}-T_{2}\right)\right] x}-\frac{\lambda^{*}}{b}, \\
& R_{e f f}^{*}=\frac{d}{b \frac{T_{1}+T_{2}}{2}+\lambda^{*}}=\frac{d}{\lambda\left(T_{e f f}^{*}\right)}=\frac{d}{\lambda_{e f f}^{*}}, \\
& T_{\text {eff }}^{*}=\frac{T_{1}+T_{2}}{2}, \quad \lambda_{e f f}^{*}=\frac{\lambda\left(T_{1}\right)+\lambda\left(T_{2}\right)}{2} .
\end{aligned}
$$

As can be seen, the temperature profile (23) is non-linear. Nevertheless, in a narrow temperature range, e. g. $T \in\left(T_{2}=255 \mathrm{~K}, T_{1}=293 \mathrm{~K}\right)$, the graph $T(x)$ will deviate only slightly from the linear profile (Fig. 3) so that a straight line can be considered as a good approximation for this case. Since the linear fit (10) is restricted to narrow temperature ranges only, the extension of the discussed solution to wider temperature ranges would be rather artificial unless the non-linear fit (15) is used (see next Chapter).

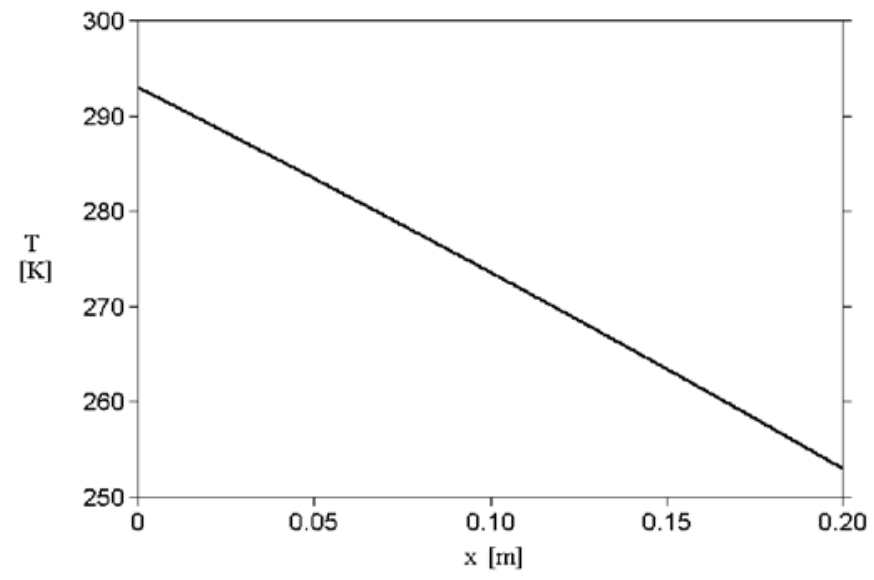

Fig. 3: Temperature profile within the foam concrete wall in a narrow temperature range.

Parameters: $d=0.2 \mathrm{~m}, T_{1}=293 \mathrm{~K}, T_{2}=253 \mathrm{~K}$, $b=1.90 \cdot 10^{-4} \mathrm{Wm}^{-1} \mathrm{~K}^{-2}, \lambda^{*}=0.0116 \mathrm{Wm}^{-1} \mathrm{~K}^{-1}$.

It is worth noting that in the case of linear dependence $\lambda(T)$ it is possible to introduce a generalised concept of thermal resistance $R_{e f f}^{*}$ using the effective constant conductivity $\lambda_{e f f}^{*}$

$$
R_{\text {eff }}^{*}=\frac{d}{\lambda_{\text {eff }}^{*}}, \quad \lambda_{\text {eff }}^{*}=\lambda\left(T_{\text {eff }}^{*}\right)=\frac{\lambda\left(T_{1}\right)+\lambda\left(T_{2}\right)}{2}
$$

This conclusion holds quite independently on temperature range.

Utilization of the thermal conductivity $\lambda\left(T_{e f f}^{*}\right)$ for the average 'surface' temperature $T_{\text {eff }}^{*}=\left(T_{1}+T_{2}\right) / 2$ can also be found in the classical work of Glaser [3] who supposed a less 
general approximation $\lambda(T)=b T$. However, using the more general function (10), one should be aware that $\lambda$ must not assume negative values $(\lambda>0)$ otherwise the corresponding solution of the system (20), (21) becomes unacceptable from the physical point of view (a flat contradiction of the second law of thermodynamics).

\subsection{Parabolic temperature dependence $\lambda(T)$}

The solution of the system (20), (21) for parabolic conductivity function (15) and the boundary conditions $T(0)=T_{1}$, $T(d)=T_{2}$ reads

$$
\begin{gathered}
q=\frac{T_{1}-T_{2}}{\frac{d}{\lambda_{0}}+\frac{\left(T_{1}-T_{0}\right)^{3}-\left(T_{2}-T_{0}\right)^{3}}{3 \frac{d}{a}}=\frac{T_{1}-T_{2}}{R_{e f f}},} \\
x=\frac{a\left(T(x)-T_{0}\right)^{3}+3 \lambda_{0}\left(T(x)-T_{0}\right)-a\left(T_{1}-T_{0}\right)^{3}}{a\left(T_{2}-T_{0}\right)^{3}+3 \lambda_{0}\left(T_{2}-T_{1}\right)-a\left(T_{1}-T_{0}\right)^{3}} d, \\
R_{\text {eff }}^{*}=\frac{d}{a \frac{\left(T_{1}-T_{0}\right)^{2}+\left(T_{1}-T_{0}\right)\left(T_{2}-T_{0}\right)+\left(T_{2}-T_{0}\right)^{2}}{3}+\lambda_{0}}= \\
=\frac{d}{\lambda_{\text {eff }}}, \\
\lambda_{\text {eff }}=\frac{1}{3}\left[\lambda\left(T_{1}\right)+\lambda\left(T_{2}\right)+\sqrt{\left(\lambda\left(T_{1}\right)-\lambda_{0}\right)\left(\lambda\left(T_{2}\right)-\lambda_{0}\right)}+\lambda_{0}\right] .
\end{gathered}
$$

Also in this case the non-linear temperature profile (28) derived in implicit form - can be encountered. The parabolic fit $(15)$ is meaningful especially in a wider temperature range $\left(T_{1} \gg T_{2}\right)$ which is just the convenient event for a full development of strong non-linearity in the $T(x)$ profile (see Fig. 4). The 'surface' temperatures $T_{1}=1500 \mathrm{~K}$ and $T_{2}=400 \mathrm{~K}$, used in Fig. 4, simulate blast-furnace thermal conditions.

Again in a steady thermal state the heat flux $q$ can be calculated using the effective thermal resistance $R_{\text {eff }}$ introduced by means of constant effective conductivity $\lambda_{\text {eff }}$ (see (30)).

\section{Conclusion}

A steady thermal state is characterised by a constant heat flux that can be determined by means of an effective thermal resistance and the 'surface' temperature difference of the wall under investigation. The expression of effective thermal resistance is dependent - besides the wall thickness $d$ - on the analytical form of $\lambda(T)$. This paper has illustrated that within the steady thermal state it is always possible to find a constant resistance (in the conventional sense) regardless of the eventual temperature dependence $\lambda(T)$

$$
R_{\text {eff }}=\frac{d}{\lambda_{\text {eff }}} .
$$

This fact has been illustrated with two particular examples, namely, for linear and parabolic functions $\lambda(T)$.

The effective conductivity $\lambda_{\text {eff }}$ for the linear $\lambda$-function (10) assumes the value of the average 'surface' conductivity

$$
\lambda_{\text {eff }}^{*}=\frac{1}{2}\left[\lambda\left(T_{1}\right)+\lambda\left(T_{2}\right)\right] .
$$

For the parabolic $\lambda$-function (15) a more complicated combination of 'surface' conductivity determines the effective value $\lambda_{\text {eff }}=\frac{1}{3}\left[\lambda\left(T_{1}\right)+\lambda\left(T_{2}\right)+\sqrt{\left(\lambda\left(T_{1}\right)-\lambda_{0}\right)\left(\lambda\left(T_{2}\right)-\lambda_{0}\right)}+\lambda_{0}\right]$

As soon as temperature dependence appears with the thermal conductivity $\lambda(\mathrm{T})$, the corresponding temperature profile $\mathrm{T}(\mathrm{x})$ inside a wall becomes non-linear. However, this non-linearity proves to be essential only for a sufficiently wide temperature range $\left(T_{1} \gg T_{2}\right)$. Such a situation can be encountered, e.g., with blast-furnace envelopes which experience extreme temperature gradients and, therefore, pronounced non-linearity of the corresponding temperature profiles must be expected. A pronounced non-linear profile (Fig. 4) caused by an extreme thermal range and the cubic conductivity function (15) has been illustrated and analytically described (28) in this paper.

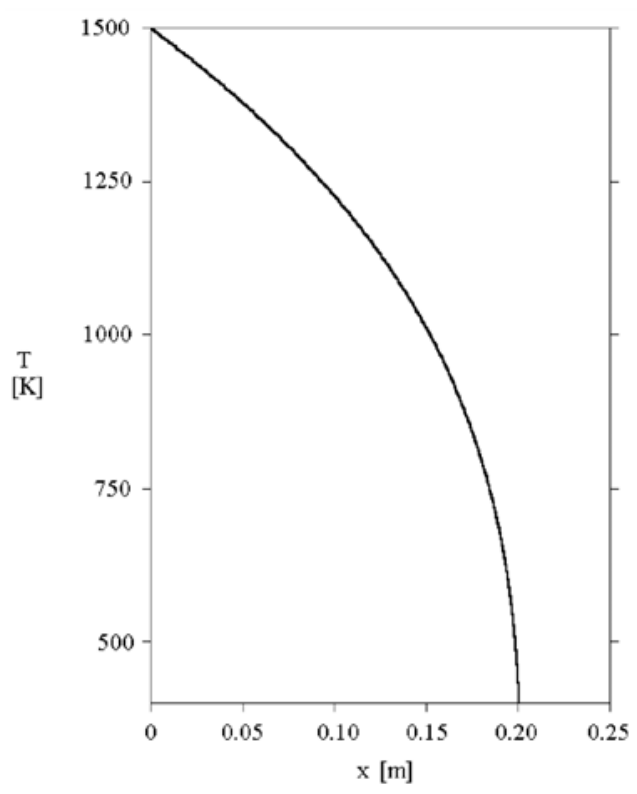

Fig. 4: Temperature profile within a foam concrete wall in a wide temperature range. Parameters: $d=0.2 \mathrm{~m}, T_{1}=1500 \mathrm{~K}$, $T_{2}=400 \mathrm{~K}, a=6 \cdot 10^{7} \mathrm{Wm}^{-1} \mathrm{~K}^{-3}, T_{0}=165 \mathrm{~K}$, $\lambda_{0}=0.0570 \mathrm{Wm}^{-1} \mathrm{~K}^{-1}$.

\section{References}

[1] Halahyja, M.: Stavebná tepelná technika. Bratislava, SVLT 1966 (in Czech)

[2] Toman, J.: Thermal conductivity of High Performance Concrete in Wide Temperature and Moisture Ranges. Acta Polytechnica, Vol. 41, No. 1/2001, pp. 8-10

[3] Glaser, H.: Influence of temperature on the diffusion of vapour through dry insulation walls. (In German), Kältetechnik, Vol. 9, No. 6/1957, pp. 158-159

Assoc. Prof. RNDr. Tomáš Ficker, DrSc.

phone: +420541147661

e-mail: fyfic@fce.vutbr.cz

Department of Physics

Professor Ing. arch. Jiří Myslín, CSc. Ing. Z. Podešvová, Ph.D. student

Department of Building Structures

University of Technology

Faculty of Civil Engineering

Žižkova 17, 66237 Brno, Czech Republic 